\title{
Diseño y aplicación de indicadores de calidad paisajística para la evaluación de atractivos turísticos en áreas rurales ${ }^{1}$
}

\author{
Arturo García Romero², \\ Manuel Antonio Serrano de la Cruz Santos-Olmo ${ }^{3}$, \\ Alberto Méndez-Méndez ${ }^{4}$ y Eduardo Salinas Chávez ${ }^{5}$
}

\section{RESUMEN}

Considerando que el turismo es una actividad que contribuye al bienestar de las comunidades rurales, se propone un método para identificar y evaluar atractivos turísticos, con base en su calidad paisajística y su viabilidad social. El método consiste en el diseño y la combinación de indicadores de tipo escénico, funcional e interpretativo para evaluar la calidad del paisaje; y en el reconocimiento, mediante entrevistas y encuestas, de la percepción que la comunidad local tiene del turismo, su interés y disposición por participar y su nivel de organización. La investigación se desarrolló en Atlautla, un municipio cercano a la Ciudad de México. Se identificaron 29 atractivos turísticos, incluyendo 7 de alta calidad paisajística y preferencia, a partir de los cuales sería viable expandir los beneficios de la actividad turística en la comunidad. No obstante que la comunidad tiene suficiente conocimiento, interés y disposición en participar, carece de una adecuada capacidad organizativa.

Palabras clave: Paisaje, indicadores de paisaje, turismo alternativo, percepción local, México.

\begin{abstract}
Considering that tourism is an activity that contributes to the well-being of rural communities, a method is proposed to identify and evaluate tourist attractions, based on their landscape quality and their social viability. The method consists in the design and combination of scenic, functional and interpretive indicators to evaluate the quality of the landscape; and in the recognition, through interviews and surveys, of the perception that the local community has of tourism, their interest and willingness to participate and their level of organization. The research was developed in Atlautla, a municipality near Mexico City. Twenty-nine tourist attractions were identified, including seven high-quality landscaping and preference, from which it would be viable to expand the benefits of tourism activity in the community. Although the community has sufficient knowledge, interest and willingness to participate, it lacks adequate organizational capacity.
\end{abstract}

Key words: Landscape, landscape indicators, alternative tourism, local perception, Mexico.

\footnotetext{
Este estudio se ha llevado a cabo en el marco del Proyecto DGAPA-PAPIIT- IN301218 de la Universidad Nacional Autónoma de México. Artículo recibido el 10 de agosto de 2017, aceptado el 17 de mayo de 2018, corregido el 12 de octubre de 2018.

Departamento de Geografía Física, Instituto de Geografía. E-mail: agromero@igg.unam.mx

Departamento de Geografía y Ordenación del Territorio, Universidad de Castilla-La Mancha (España). E-mail: manuel.serranocruz@uclm.es

Facultad de Estudios Superiores Zaragoza. E-mail: albmen@unam.mx

5 Universidade Federal de Mato Grosso do Sul (Brasil). E-mail: esalinasc@yahoo.com
} 
En la actualidad el turismo es considerado como una actividad alternativa para fomentar la economía de las áreas rurales alrededor del mundo. Con frecuencia, la mayor atención está puesta en las áreas rurales periféricas a las ciudades, que aún conservan un patrimonio natural, paisajístico y cultural destacado (Carneiro et al., 2015; Fernández Robin et al., 2015), y donde se pretende impulsar el bienestar de las comunidades locales, incentivando el uso sustentable de los recursos naturales, los paisajes y el patrimonio cultural (Salinas et al., 2008; Castillo et al., 2009). Inclusive, en muchos países en desarrollo la actividad turística se ha convertido en una opción complementaria para enfrentar la caída de los medios tradicionales de subsistencia, la pobreza y la emigración de los sectores más jóvenes de la población rural.

Sin embargo, los proyectos turísticos surgen, con frecuencia, de forma espontánea y sin planificación, con base en una demanda creciente de visitantes y una fuerte sobrecarga en torno a escasos productos, en ocasiones ilegítimos y de baja calidad, que son tratados como atractivos exclusivamente comercializables. Por desconocimiento o falta de interés, en muchos casos se dejan de lado importantes recursos del paisaje que pueden contribuir al desarrollo de propuestas integrales de mayor calidad paisajística, como son la calidad ambiental y ecológica de los componentes naturales, el apego social hacia los elementos tangibles e intangibles de la historia y la evolución del uso del suelo, el arte y la música inspirados en el paisaje o el valor científico derivado de la relación naturaleza-sociedad (Picazo, 2012; Cebrián-Abellán, 2013).

El uso de indicadores de calidad del paisaje puede ser de gran utilidad para enfrentar esta situación, pues representan una importante herramienta de evaluación y monitoreo de diferentes realidades territoriales, facilitando la identificación de recursos, cualidades y problemáticas, especialmente útiles para su adecuada valoración y gestión (Bruni, 2016). Recientemente, el uso de indicadores del paisaje ha experimentado un notable incremento (Uuemaa et al., 2013), con resultados exitosos en múltiples aplicaciones, como el análisis de las relaciones naturaleza-sociedad y la evaluación de las transformaciones territoriales (Gottero \& Cassatella, 2017), con especial atención al potencial del uso del suelo y los riesgos ambientales asociados (Ceballos \& López-Blanco, 2003). Los indicadores de calidad del paisaje son parámetros cuantitativos o cualitativos que proporcionan una información clara (sin ambigüedad), objetiva (con un criterio de selección), relevante (para los tomadores de decisiones), consistente (de solidez analítica), de fácil medición (buena relación costo-beneficio) e integrada (a partir de un modelo conceptual) (OECD, 2003; Serrano, 2008; Nogué et al., 2009; Cassatella \& Peano, 2011), cuyo significado para el turismo está en relación con su contribución a la singularidad del destino, su imagen, su identidad y su atractivo (Zuluaga, 2006; Castillo et al., 2009; Fernández Robin, 2015).

Sin embargo, estas útiles herramientas de diagnosis paisajística encierran también un conjunto de problemas que deben ser superados. Entre ellos, se destacan que su construcción es complicada, al requerir de la exigencia de un modo crítico de reflexionar, de apropiarse y reconstruir la realidad (Gutiérrez, 2009); la dificultad de su diseño, frecuentemente supeditado a la superposición de observaciones ecológicas o puramente estéticas (Fry et al., 2009); la fundamentación de la selección de elementos y criterios de evaluación, habitualmente recogidos en una amplia variedad de categorías que introducen la sobreestimación de determinados valores (Cassatella \& Peano, 2011; Sowińska-Świerkosz \& Chmielewski, 2016); o la búsqueda no resuelta de la universalidad en su aplicación a diferentes escalas y regiones. En ocasiones, la falta de equilibrio motivado al otorgar un excesivo énfasis a determinados elementos, como la calidad ecológica y ambiental 
- la agricultura y los usos del suelo, limitan su empleo e impiden su implementación efectiva en los procesos de evaluación (Cassatella \& Peano, 2011; Sowińska-Świerkosz \& Chmielewski, 2016).

Siendo el paisaje un recurso con un alto potencial para la actividad turística, uno de los temas más debatidos es el relacionado con la evaluación de la importancia y magnitud de dicho potencial. Numerosos estudios lo hacen desde el campo de las ciencias sociales, desde donde, con frecuencia, las principales críticas al uso de indicadores como herramienta de valoración apuntan a que el esfuerzo realizado para dotar a los estudios de objetividad y consistencia puede tener efectos negativos (Muñoz-Pedreros, 2004), al limitar la aprehensión de los aspectos (personales y sociales) relacionados con la apreciación perceptiva de los individuos (Alonso, 2009).

Frente a estas posturas, diversos trabajos han probado la utilidad del uso de indicadores ante algunos problemas del sistema turístico, como es el caso de la homogenización de los destinos, la idealización de determinados atractivos sin atender a la legitimidad y calidad de los recursos (Leader-Elliott, 2005; Geronta, 2016), y la estacionalidad de la actividad ocasionada por una escasa diversificación territorial de los productos (Fernández-Robin et al., 2015). Si partimos de considerar que la imagen mental que se produce al observar un determinado paisaje es la que se pretende poner en valor a la hora de generar un producto turístico, este estudio se interesa por el uso de indicadores para la valoración del paisaje, en tanto referente real de la imagen percibida. Esto se sustenta en la idea, generalmente aceptada, de que el paisaje es el resultado de la combinación de elementos físicos, biológicos y humanos (Muñoz-Pedreros, 2004; Serrano, 2008; Nogué et al., 2009), contenidos ambientales que pueden cubrir los significados psicológicos de la imagen visual que es percibida por el observador y, por lo tanto, de ellos depende el interés por el paisaje como recurso turístico (Cebrián-Abellán, 2013).

Por otra parte, se ha visto que los diagnósticos son más efectivos cuando se realizan desde los intereses de la propia comunidad local, la cual es considerada como la mejor conocedora de los atractivos turísticos de su entorno. La participación de la comunidad proporciona una visión más real para el diseño de las propuestas, y conduce a un proceso de toma de decisiones sostenible y coherente en torno a objetivos comunes y acuerdos compartidos, evitando entornos conflictivos durante las etapas posteriores de diseño, planificación y gestión (Geronta, 2016). El aporte del turismo al desarrollo local será, por tanto, más efectivo, cuanto más endógeno sea el diseño de los productos turísticos en cuestión (Barbini, 2005; OECD, 2003).

El objetivo de este estudio es exponer y aplicar un método basado en la combinación de indicadores de calidad del paisaje y en la percepción de la comunidad local, válido para el diseño de productos turísticos alternativos, auténticos y viables desde el punto de vista social, que contribuyan al desarrollo económico de las áreas rurales, fundamentado en el desarrollo de un turismo integrado y sostenible, que respete y mantenga los principales valores naturales y culturales de las áreas involucradas.

La investigación se llevó a cabo en el municipio de Atlautla (1.900 a 5.426 msnm; 167,6km²), localizado en la vertiente occidental del volcán Popocatépetl, a $75 \mathrm{~km}$ al SE de la Ciudad de México (Figura $N^{\circ}$ 1). La mayor parte de la población ( 30.000 habitantes) se distribuye en cuatro localidades, Atlautla de Victoria, San Juan Tehuixtitlán, San Juan Tepecoculco y San Andrés Tlalamac, cuyos nombres reflejan la riqueza cultural heredada de su origen mestizo (azteca y español) (INA- 
FED, 2018). Como es común de los pueblos cercanos a la capital del país, el valor cultural de los pueblos de Atlautla se asienta en su historia, tradiciones, gastronomía y monumentos históricos.

Por otra parte, al ser el volcán Popocatépetl un elemento icónico de relevancia nacional e internacional, los recursos naturales del municipio adquieren especial valor. El desnivel altitudinal supera los $3.000 \mathrm{~m}$ y permite una destacada diversidad ambiental, con 4 unidades de relieve - alta montaña, ladera media, piedemonte piroclástico y piedemonte lávico -, y 5 pisos bioclimáticos, incluyendo varios tipos de bosque templado y pastizales alpinos; el término Atlautla, de la lengua nahualtl hablada por los aztecas, significa "lugar entre barrancos", en alusión al relieve agreste que caracteriza a las vertientes del volcán (Figura $N^{\circ} 2$ ).

Figura $\mathrm{N}^{\circ} 1$.

Ubicación del municipio de Atlautla en la vertiente occidental del volcán Popocatépetl, centro de México

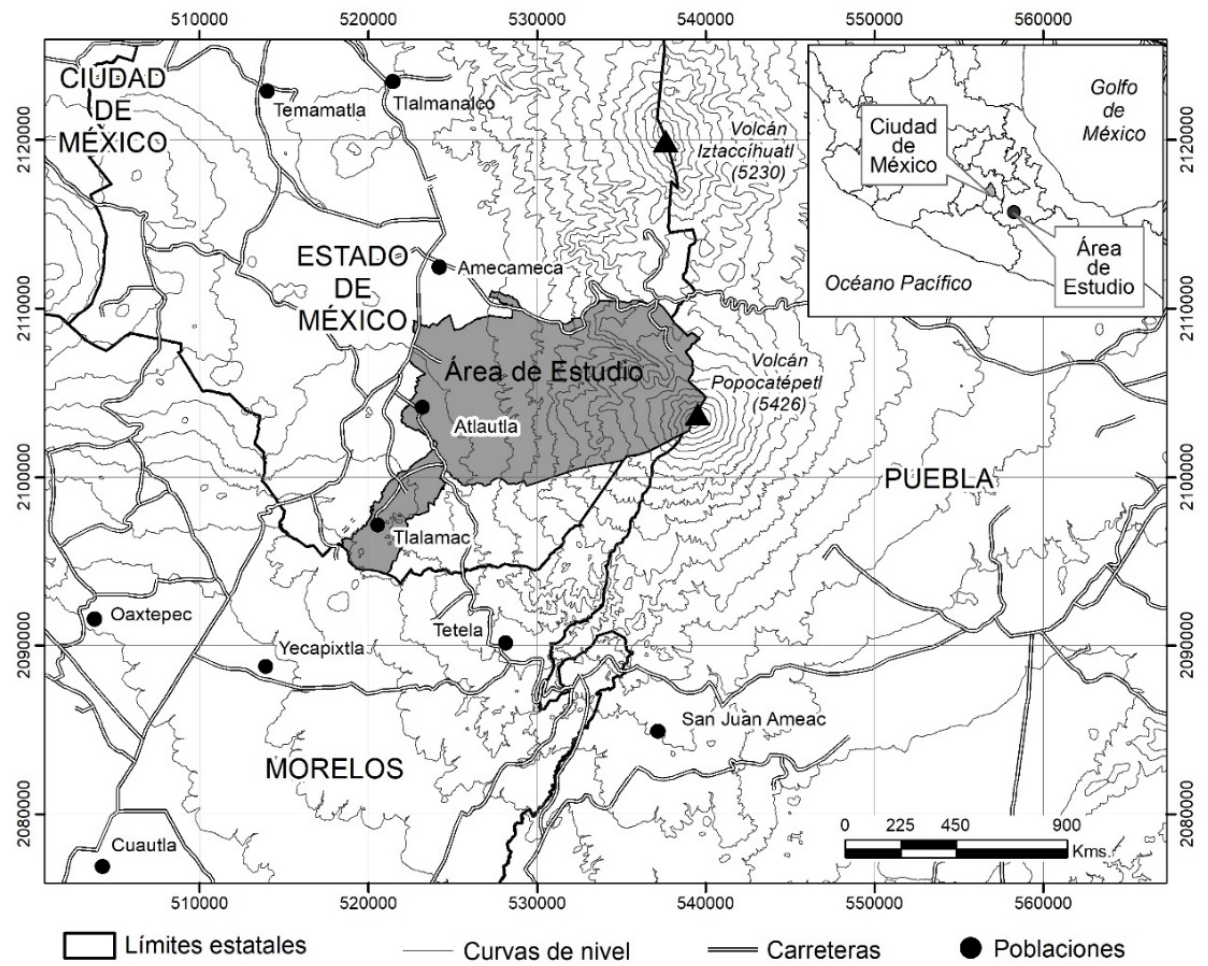

Fuente: Elaboración propia.

No obstante que el municipio se encuentra próximo a importantes centros de recreación y esparcimiento en espacios forestales y de alta montaña, no ha logrado insertarse en el sistema turístico regional. En la actualidad, el uso del suelo predominante es forestal $(45,2 \%)$ y agrícola $(30,3 \%)$ (INEGI, 2009), reflejando una economía centrada en el cultivo, principalmente de maíz, frijol, hortalizas y frutales. Sin embargo, la superficie municipal está repartida en propiedad privada (40,6 $\%)$, bienes comunales $(50,1 \%)$ y ejidos $(9,3 \%)$, situación que da lugar a conflictos relacionados con 
el uso de la tierra, el decrecimiento de la producción agrícola y el comercio, el incremento de la pobreza y la emigración de la población joven.

Frente a esta situación, la comunidad está interesada en desarrollar el turismo como una actividad complementaria que le permita incrementar su bienestar económico y social, incentivando el uso sustentable de los recursos naturales y del patrimonio cultural. Las expectativas son claras en cuanto al interés por atender la demanda del turismo regional, interesado por actividades recreativas en ambientes naturales, la cultura y la gastronomía de la región.

\section{Metodología}

El método presentado se compone de tres etapas: 1) Identificación y caracterización de los atractivos turísticos, realizadas con el apoyo de miembros de la comunidad mediante entrevistas; 2) Diseño y aplicación de indicadores para evaluar la calidad paisajística de los atractivos turísticos, con base en criterios objetivos y mensurables que permitan una valoración directa de los recursos geomorfológicos, bióticos y culturales, (OECD, 2003; Pralong, 2005; Reynard et al., 2007; Nogué et al., 2009); y 3) Diagnóstico de la percepción local del turismo, mediante encuestas y entrevistas, para identificar la disposición y preferencias de la comunidad por participar en la actividad turística.

\section{Identificación y caracterización de los atractivos turísticos}

Esta etapa consiste en la realización de diversos recorridos con miembros de la comunidad, como fuente primaria de recogida de información para la elaboración del inventario de los atractivos turísticos con potencial para el turismo. Consta de dos fases, en primer lugar la información es obtenida mediante entrevistas a informantes clave, incluyendo líderes de la comunidad (representantes del gobierno y de las distintas formas de propiedad de la tierra, sacerdotes, otros líderes espirituales, etc.), académicos radicados en la localidad, e informantes relacionados con la actividad turística (en este caso, guías de turismo, productores de miel y comerciantes dedicados a la venta de alimentos); posteriormente, en una segunda fase tiene lugar el levantamiento de encuestas que permiten la recogida de información de sectores más amplios de la comunidad.

La identificación de los atractivos se hace sobre la base de la diversidad paisajística del territorio municipal, bajo la premisa de considerar al paisaje como un concepto accesible y comprensible para amplios sectores de la sociedad y que además tiene un fuerte componente de identidad colectiva y de sensibilización con la transformación del territorio (Dosso, 2011; Picazo, 2012).

\section{Diseño y aplicación de indicadores para evaluar la calidad paisajística de los atractivos turísticos}

Para la formulación de los indicadores de calidad paisajística de los atractivos se propone la adaptación del modelo conceptual de G. Bertrand (Bertrand \& Bertrand, 2006; Muñoz, 1998), según la cual, la calidad de un atractivo turístico reside en distintos recursos que pertenecen a tres componentes fundamentales del paisaje: geomorfológico, biótico y cultural. Para evaluar la calidad de estos recursos proponemos el uso de un conjunto de indicadores, relativos a tres criterios - escénico, funcional e interpretativo -, donde cada uno de ellos responde a un aspecto de 
relevancia en la valoración de los atractivos turísticos (ver incisos a - c y Cuadro $\left.N^{\circ} 1\right)$. Aunque de manera general los criterios tienen cierto grado de analogía con los propuestos por diversos autores, al final corresponden a un diseño propio, siguiendo un esquema estructurado, de tal forma que la consideración del conjunto de criterios e indicadores permite una evaluación integral del potencial del paisaje como recurso para el turismo (Muñoz-Pedreros, 2004).

a) Criterio escénico ( 9 indicadores). Valora el agrado perceptual que se tiene por la belleza del paisaje. Interesa la cantidad y calidad de los componentes visuales del paisaje; el contraste entre las formas, colores y perspectiva que resultan del conjunto de ellos; así como la existencia de componentes excepcionales de gran valor, como son los cuerpos de agua (Zuluaga, 2006; Serrano, 2008; Knudsen et al., 2013; Kalivoda et al., 2014). Se consideran, tanto el valor escénico del sitio, como el valor que resulta de la amplitud panorámica que permita las vistas a distancia, tanto en sitios puntuales como en trayectos longitudinales.

b) Criterio funcional (14 indicadores). Valora la estabilidad geomorfológica, el grado de conservación de los componentes bióticos y la historia, productividad, sustentabilidad, accesibilidad y equipamiento asociados a los componentes culturales del paisaje (Pralong, 2005; Salinas et al., 2008; Nogué et al., 2009). Se considera además la importancia del patrimonio material (p.e. en infraestructura) e inmaterial (p.e. espiritual e histórico-cultural) asociado al sitio, así como la originalidad y relevancia de la relación entre los componentes del paisaje y la importancia de alguno de ellos para el resguardo y protección de los otros.

c) Criterio interpretativo (6 indicadores). Se enfoca en las características didácticas y de ejemplaridad del sitio, como exponente de la singularidad y representatividad de alguno de los componentes del paisaje, y de su importancia para facilitar explicaciones didácticas y despertar el interés por estudios académicos para la comprensión del paisaje (Reynard et al., 2007). Interesa el nivel de alcance del sitio, tanto asociado a la calidad propia de alguno de sus componentes, como a la configuración general del paisaje.

\section{Cuadro $\mathrm{N}^{\circ} 1$}

Indicadores para valorar la calidad paisajística de los atractivos turísticos en áreas rurales. Se indican los componentes del paisaje y los criterios de valoración considerados

\begin{tabular}{|c|c|c|c|}
\hline \multirow{2}{*}{$\begin{array}{l}\text { Criterios de } \\
\text { valoración }\end{array}$} & \multicolumn{3}{|c|}{ Indicadores de calidad por componente del paisaje } \\
\hline & Biótico & Geomorfológico & Cultural \\
\hline Escénico & Tipo de vegetación & $\begin{array}{l}\text { Calidad escénica intrínseca } \\
\text { Desnivel topográfico } \\
\text { Presencia de cuerpos de } \\
\text { agua } \\
\text { Amplitud panorámica } \\
\text { Variedad paisajística pano- } \\
\text { rámica } \\
\text { Longitud del tramo con pa- } \\
\text { norámicas }\end{array}$ & $\begin{array}{l}\text { Calidad escénica de la } \\
\text { superficie cultivada } \\
\text { Calidad escénica del } \\
\text { asentamiento humano }\end{array}$ \\
\hline
\end{tabular}


Continuación Cuadro N¹

\begin{tabular}{|c|c|c|c|}
\hline \multirow{2}{*}{$\begin{array}{l}\text { Criterios de } \\
\text { valoración }\end{array}$} & \multicolumn{3}{|c|}{ Indicadores de calidad por componente del paisaje } \\
\hline & Biótico & Geomorfológico & Cultural \\
\hline Funcional & $\begin{array}{l}\text { Estado de conserva- } \\
\text { ción } \\
\text { Cercanía a Áreas Na- } \\
\text { turales Protegidas } \\
\text { Servicios ambientales }\end{array}$ & $\begin{array}{l}\text { Integridad } \\
\text { Asociación con la conserva- } \\
\text { ción del ecosistema } \\
\text { Asociación con rasgos cul- } \\
\text { turales de valor paisajístico }\end{array}$ & $\begin{array}{l}\text { Trascendencia religiosa } \\
\text { o mística } \\
\text { Trascendencia histórica } \\
\text { Trascendencia artística } \\
\text { y cultural } \\
\text { Productos económicos } \\
\text { Nivel de alcance del } \\
\text { atractivo } \\
\text { Equipamiento } \\
\text { Tipo de acceso } \\
\text { Distancia al sitio }\end{array}$ \\
\hline Interpretativo & $\begin{array}{l}\text { Representatividad } \\
\text { Singularidad }\end{array}$ & $\begin{array}{l}\text { Representatividad } \\
\text { Singularidad }\end{array}$ & $\begin{array}{l}\text { Representatividad } \\
\text { Singularidad }\end{array}$ \\
\hline
\end{tabular}

Fuente: Elaboración propia.

Los 29 indicadores propuestos para este estudio son objetivos y mensurables, permitiendo establecer una base de datos altamente explicativa y sistemática, con la cual se determina la calidad de los atractivos con mayores alcances hacia objetivos prácticos de aprovechamiento turístico (Salinas et al., 2008; Serrano, 2008; Nogué et al., 2009). Todos ellos son seleccionados y evaluados de forma directa mediante la información reunida en el trabajo de campo, y con el apoyo de informantes locales relacionados con la actividad turística.

Cada uno de los atractivos turísticos estudiados es caracterizado mediante una ficha de campo que contiene los indicadores propuestos para valorar su calidad. Cada indicador se califica en una escala de cinco niveles de calidad $(0,0,25,0,5,0,75$ y 1$)$, siendo 1 la máxima calidad que un indicador puede obtener. Las fichas son cumplimentadas en el campo y los resultados se integran en una base de datos. La calidad del atractivo turístico (Cat) se calcula finalmente según la fórmula siguiente:

$$
C_{a t}=1.61(V b)+0.88(V g)+0.81(V c)
$$

Dónde: (Cat) Calidad del atractivo turístico; $(\mathrm{Vb})$ suma de los valores de calidad dentro del componente biótico; $(\mathrm{Vg})$ suma de los valores de calidad dentro del componente geomorfológico; (Vc) suma de los valores de calidad dentro del componente cultural; 1,61, 0,88 y 0,81 son factores de ponderación para evitar el sesgo derivado del hecho que la cantidad de indicadores varía entre los tres componentes del paisaje.

Los atractivos se clasifican con base en el valor de su Cat con respecto al máximo posible $\left(C_{\text {max }}=29\right)$. Se consideran tres categorías: Bajo (menos de 9,67), Medio $(9,67-19,36)$ y Alto $(19,37$ - 29), lo cual permite homogenizar los resultados y facilitar su interpretación. 


\section{Diagnóstico de la percepción local del turismo}

La viabilidad social del turismo depende, entre otros aspectos, de la presencia de una cultura local de desarrollo, misma que puede ser revelada a través de la aplicación de encuestas aleatorias y entrevistas a miembros de la comunidad, incluyendo a informantes clave (líderes políticos y religiosos, y representantes de los diferentes niveles de gobierno), cuya opinión puede resultar de interés para la interpretación y discusión de los resultados. La intención primordial es rescatar opiniones y percepciones en relación a tres dimensiones del capital social (Barbini 2005, 2008) (Cuadro $N^{\circ}$ 2).

a) Percepción local del turismo. Se refiere a la imagen percibida por los actores locales sobre la localidad y su gente, el turismo y los turistas, la gestión local y su participación. Dada la posibilidad de que la población no conozca todos los atractivos turísticos identificados, es conveniente que el estudio de preferencia se realice de forma indirecta, por ejemplo, a partir de identificar las actividades turísticas preferidas por la población local, y de observar su distribución al interior de los atractivos evaluados.

b) Interés y disposición por participar. Esta dimensión del capital social permite evaluar las actitudes participativas, creativas e innovadoras de los distintos actores sociales, y de la cultura local en su conjunto. Asimismo, facilita la visualización de la diversidad de las actividades turísticas potenciales.

c) Nivel de organización. Se refiere al grado de integración y organización de la comunidad, con especial atención en la participación activa de líderes locales con capacidad de convocar y movilizar a los diferentes actores sociales, bajo el entendido de que un buen nivel de organización favorece la capacidad de la comunidad para enfrentar desafíos diversos.

Cuadro $\mathrm{N}^{\circ} 2$

Indicadores de la percepción local del turismo

\begin{tabular}{|l|l|}
\hline $\begin{array}{l}\text { Dimensión del capital } \\
\text { social }\end{array}$ & \multicolumn{1}{|c|}{ Indicador de la viabilidad social del turismo } \\
\hline $\begin{array}{l}\text { Percepción local del turis- } \\
\text { mo }\end{array}$ & Conocimiento de la existencia de atractivos turísticos \\
\hline & Conocimiento preciso de la diversidad de atractivos turísticos \\
\hline & $\begin{array}{l}\text { Conocimiento de la finalidad por la que los atractivos turísticos } \\
\text { son visitados }\end{array}$ \\
\hline & Conocimiento del flujo de visitantes en la comunidad \\
\hline $\begin{array}{l}\text { Interés y disposición por } \\
\text { participar }\end{array}$ & $\begin{array}{l}\text { Suficiencia de los ingresos económicos que perciben por su acti- } \\
\text { vidad actual }\end{array}$ \\
\hline & Percepción de los beneficioniontantes que pueden esperarse del turismo \\
\hline
\end{tabular}


Continuación Cuadro $N^{\circ} 2$

\begin{tabular}{|l|l|}
\hline $\begin{array}{l}\text { Dimensión del capital } \\
\text { social }\end{array}$ & \multicolumn{1}{c|}{ Indicador de la viabilidad social del turismo } \\
\hline & Grado de interés por participar en proyectos de turismo \\
\hline & $\begin{array}{l}\text { Preferencia por participar en determinadas actividades relaciona- } \\
\text { das con el turismo }\end{array}$ \\
\hline Nivel de organización & $\begin{array}{l}\text { Nivel de unión y colaboración colectiva en torno a objetivos co- } \\
\text { munes }\end{array}$ \\
\hline & Presencia de líderes sociales al interior de la comunidad \\
\hline & Opinión del trabajo realizado por los líderes \\
\hline & Presencia de conflictos entre actores y grupos sociales \\
\hline
\end{tabular}

Fuente: elaboración propia.

\section{Síntesis de calidad de los atractivos turísticos y propuesta de desarrollo}

A partir de los resultados obtenidos es posible generar una lista de actividades turísticas preferidas por la comunidad para participar en caso de un eventual proyecto de desarrollo turístico en el municipio. El grado de preferencia por una cierta actividad se puede obtener, por ejemplo, en términos de la proporción de votos recibidos por dicha actividad con respecto al total de votos recibidos por el conjunto de actividades. Finalmente, la calidad paisajística de los atractivos turísticos se obtiene de la interpretación comparada entre, por una parte, la calidad paisajística intrínseca de cada atractivo, y por otra su nivel de preferencia por parte de la comunidad.

\section{Inventario y valoración de los atractivos turísticos}

La combinación de las características geomorfológicas, bióticas y culturales de Atlautla favorece una alta diversidad paisajística. Con base en 22 entrevistas y diversos recorridos con informantes clave se obtuvo una cartera de 29 atractivos con potencial para el desarrollo del turismo (Figura $\mathrm{N}^{\circ}$ 2).

La evaluación de la calidad de los atractivos turísticos (Cat) permitió conocer que los componentes bióticos del paisaje son los que más contribuyen a la Cat $(=4,90)$, seguidos por los componentes geomorfológicos $(=3,60)$ y los culturales $(=2,43)$ (Cuadro $N^{\circ} 3$ ). Los Arenales del Popocatépetl son el atractivo con mayor calidad paisajística $(C a t=20,38)$, sobre todo por la excepcionalidad de sus recursos bióticos y geomorfológicos $(V b=9,26$ y $V g=7,48)$, en tanto que 16 atractivos $(55,2 \%$ del total) obtuvieron un valor medio y $12(41,4 \%)$ tuvieron valor bajo. Otros atractivos bien valorados en alguno de sus componentes paisajísticos fueron la Ruta al Santuario de la mariposa monarca $(V b=9,26)$ que constituye un nicho de alto valor ecológico a nivel mundial, la Ruta al centro ceremonial Xochiquía $(V g=6,82)$ que posibilita al turista participar en ceremonias religiosas de origen prehispánico, así como la Parroquia de San Miguel Arcángel y la Capilla de 
San Juan Bautista $\left(V_{c}=6,08\right)$, edificaciones religiosas del siglo XVII con elementos prehispánicos en fachadas y capiteles (Figura $\mathrm{N}^{\circ} 2$ y Cuadro $\mathrm{N}^{\circ} 3$ ).

\section{Cuadro $\mathrm{N}^{\circ} 3$}

Inventario y evaluación de la calidad de los atractivos turísticos. Se muestra un extracto con 5 de los 29 atractivos turísticos identificados. $V b=$ Valor biótico; $V g=V a l o r$ geomorfológico; $V c=$ Valor cultural;

Cat $=$ Calidad del atractivo turístico (Bajo, Medio, Alto)

\begin{tabular}{|l|l|c|c|c|c|c|}
\hline Nombre del atractivo & \multicolumn{1}{|c|}{ Descripción } & Vb & Vg & Vc & Cat & $\begin{array}{c}\text { Cali- } \\
\text { dad }\end{array}$ \\
\hline $\begin{array}{l}\text { Arenales del } \\
\text { Popocatépetl }\end{array}$ & $\begin{array}{l}\text { Cumbres cubiertas por mate- } \\
\text { riales volcánicos recientes }\end{array}$ & 9,26 & 7,48 & 3,65 & 20,38 & Alta \\
\hline Capilla San Juan Bautista & $\begin{array}{l}\text { Vestigios combinados de } \\
\text { pirámide prehispánica y ex } \\
\text { convento colonial }\end{array}$ & 0,81 & 2,86 & 6,08 & 9,74 & Media \\
\hline $\begin{array}{l}\text { Parroquia San Miguel } \\
\text { Arcángel }\end{array}$ & Templo católico del S. XVII & 0,40 & 2,86 & 6,08 & 9,34 & Baja \\
\hline Ruta mariposa monarca & $\begin{array}{l}\text { Trayecto en vehículo, caballo } \\
\text { o bicicleta hacia el santuario }\end{array}$ & 9,26 & 4,84 & 1,82 & 15,92 & Media \\
\hline $\begin{array}{l}\text { Ruta al centro ceremonial } \\
\text { Xochiquia }\end{array}$ & $\begin{array}{l}\text { Trayecto a través de paisajes } \\
\text { volcánicos y miradores natu- } \\
\text { rales }\end{array}$ & 8,86 & 6,82 & 2,03 & 17,70 & Media \\
\hline Máximo esperado & \multicolumn{7}{|l|}{9,67} & 9,67 & 9,67 & 29,00 & \\
\hline
\end{tabular}

Fuente: Elaboración propia.

\section{Percepción del turismo por parte de la comunidad local}

La percepción de la comunidad respecto al desarrollo de proyectos turísticos se obtuvo mediante la aplicación de 128 encuestas aleatorias, incluyendo hombres $(57,0$ "\%) y mujeres $(43,0$ "\%) con edades entre 18 y 60 años, y predominio de trabajadores del campo $(31,0$ "\%), seguidos por estudiantes $(25,0 \%)$, amas de casa $(13,0 \%)$, comerciantes $(9,0 \%)$, empleados $(8,0 " \%)$, oficios $(8,0$ $\%)$ y profesionales $(5,0 \%)$.

Frente a los bajos niveles de satisfacción socio-económica que la población percibe por sus actividades actuales ( 46,0 \% afirma que son bajos, el 49,0 "\% medianos y $5,0 \%$ altos), existe una clara percepción del potencial turístico de la comunidad, ya que el 87,0 "\% de la muestra reconoce la existencia de atractivos turísticos con suficiente potencial para obtener mejoras en los niveles de bienestar.

La percepción que la población tiene de los visitantes es un factor clave para el desarrollo turístico local. Es muy positivo que una proporción muy alta de la muestra reconoció que la presencia de los turistas es necesaria (87,5\%). La prueba estadística de Chi cuadrada demostró que la relación entre dicha necesidad y el gusto por la llegada de turistas fue muy significativa $\left(X^{2}=\right.$ 
40,507; sig.= 0,001). Las mayores frecuencias recayeron en la población que considera que la llegada de turistas es a la vez necesaria y agradable $\left(87,8^{\prime \prime} \%\right)$ (Cuadro $\left.N^{\circ} 4\right)$.

Cuadro $\mathrm{N}^{\circ} 4$

Relación entre la necesidad y el gusto por la llegada de turistas

\begin{tabular}{|l|c|c|c|c|}
\hline \multirow{2}{*}{$\begin{array}{l}\text { Gusto por la llegada del } \\
\text { turista }\end{array}$} & \multicolumn{4}{|c|}{ Necesidad por la llegada del turista } \\
\cline { 2 - 5 } & Indistinta (\%) & Necesaria (\%) & No necesaria (\%) & Total (\%) \\
\hline Agradable & 62,5 & 87,8 & 66,7 & 84,8 \\
\hline Desagradable & 0 & 0 & 33,3 & 1,8 \\
\hline Indistinta & 37,5 & 12,2 & 0 & 13,4 \\
\hline
\end{tabular}

Fuente: Elaboración propia.

\section{Interés y disposición para participar}

A partir de 341 opiniones vertidas entre los encuestados, la comunidad mostró un interés general para participar como guías o instructores en 17 distintas actividades turísticas, con una clara preferencia por las actividades en sitios naturales, donde la comunidad reconoce una mayor calidad de los atractivos. Así, las opciones más frecuentemente seleccionadas fueron la observación de montañas, cañadas y parajes (10,9"\%), el senderismo interpretativo $(8,5 \%)$, las cabalgatas y el rescate de animales y plantas $(8,2 \%)$, los talleres de educación ambiental $(7,9)$ y la escalada y rappel $(7,6)$ (Cuadro $\left.N^{\circ} 6\right)$.

\section{Nivel de organización de la comunidad}

La mayor parte de la comunidad considera que la unión y la colaboración entre los habitantes van desde regulares $(54,0 \%)$ a malas $(31,0 \%)$, reconociendo $(72,0 \%)$ la existencia de liderazgos individuales y de grupo, cuyo trabajo en la comunidad es percibido como regular (60,0"\%). El cruce de opiniones entre las relaciones al interior de la comunidad y la valoración del trabajo de los líderes muestra un resultado heterogéneo (Cuadro $N^{\circ} 5$ ), ya que la mayoría de las combinaciones ubica como regular a ambos indicadores $(79,2 \%$ ), aunque también se observó una alta frecuencia de las combinaciones "relaciones buenas-resultados buenos" (57"\%) y "relaciones malas-resultados buenos" (52,4"\%). Por otro lado, la existencia de conflictos internos, individuales y grupales, fue identificada por casi la mitad $(48,0 \%)$, mientras que el restante 52,0 \% $\%$ indica que desconoce este fenómeno.

Cuadro $\mathrm{N}^{\circ} 5$

Colaboración en la comunidad y opinión hacia los líderes

\begin{tabular}{|l|c|c|c|c|}
\hline \multirow{2}{*}{$\begin{array}{l}\text { Resultados de los } \\
\text { dirigentes }\end{array}$} & \multicolumn{4}{|c|}{ Colaboración en la comunidad } \\
\cline { 2 - 5 } & Buena (\%) & Mala (\%) & Regular (\%) & Total (\%) \\
\hline Buenos & 57,1 & 28,6 & 13,2 & 23,9 \\
\hline Malos & 0 & 19,0 & 7,5 & 9,1 \\
\hline Regulares & 42,9 & 52,4 & 79,2 & 67,0 \\
\hline
\end{tabular}

Fuente: elaboración propia. 


\section{Identificación de los atractivos con alta calidad paisajística y preferencia para el desarrollo del turismo}

La distribución de las 17 actividades turísticas propuestas por la comunidad al interior de los distintos atractivos turísticos revela que todos ellos incluyen recursos para el desarrollo de diversas actividades, e incluso varios atractivos mostraron tener más de una función dentro de la dinámica turística (Cuadro $N^{\circ} 6$ ). De un total de 29 atractivos, siete son de alta calidad y preferencia, diez son de alta calidad y baja preferencia, dos de baja calidad y alta preferencia, y diez de baja calidad y preferencia (Figura $\mathrm{N}^{\circ} 2$ ). El primer grupo debe ser considerado como de atractivos focales, mediante los cuales insertar a otros atractivos de menor calidad paisajística, con la intención de expandir el desarrollo de la actividad turística hacia sectores más amplios dentro de la comunidad. Tres de los atractivos turísticos con mayor calidad (Arenales del Popocatépetl, Mirador arenales y Paraje La Joya Redonda) se ubican en el sector alto de la vertiente del volcán. Algunos trayectos de montaña también son reconocidos por la comunidad como atractivos turísticos de alta calidad paisajística (Ruta de la mariposa monarca, Rutas del agua 2 y 3 y Ruta al centro ceremonial Xochiquía), ya que sintetizan la calidad visual de los recursos con el apego a la tierra y sus raíces históricas.

\section{Cuadro $\mathrm{N}^{\circ} 6$}

Evaluación de los atractivos turísticos (extracto con 9 de 29 atractivos) a partir de las preferencias por parte de la comunidad local. Se muestra la proporción de votos obtenidos por las 17 actividades propuestas por la comunidad (a-q) en relación al total (341 votos). En la última columna se incluye la calidad de los atractivos turísticos (Cat).

\begin{tabular}{|c|c|c|c|c|c|c|c|c|c|c|c|c|c|c|c|c|c|c|c|c|c|}
\hline $\begin{array}{l}\text { Evaluación } \\
\text { del atractivo } \\
\text { turístico }\end{array}$ & $\begin{array}{l}\text { Nombre del } \\
\text { atractivo turístico }\end{array}$ & 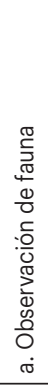 & 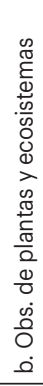 & 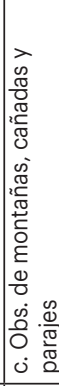 & $\begin{array}{l}\frac{\pi}{0} \\
\pi \\
\frac{\pi}{\pi} \\
\frac{0}{0} \\
\frac{0}{\pi} \\
0 \\
0 \\
0\end{array}$ & 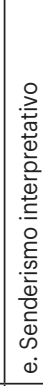 & 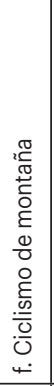 & 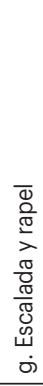 & 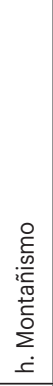 & 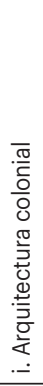 & 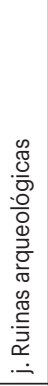 & 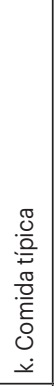 & 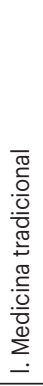 & 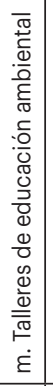 & 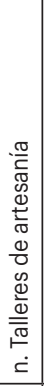 & 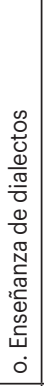 & 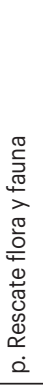 & 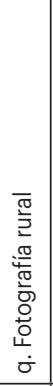 & 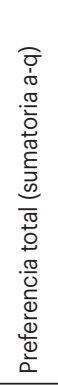 & 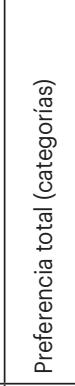 & 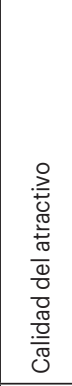 \\
\hline \multirow{3}{*}{$\begin{array}{l}\text { Alta Calidad y } \\
\text { Preferencia }\end{array}$} & $\begin{array}{l}\text { Arenales del } \\
\text { Popocatépetl }\end{array}$ & 6,2 & 5,3 & 10,9 & 0 & 8,5 & 0 & 0 & 3,8 & 0 & 0 & 0 & 0 & 0 & 0 & 0 & 0 & 5,0 & 39,6 & Medio & Alta \\
\hline & Paraje Joya Redonda & 6,2 & 5,3 & 10,9 & 0 & 8,5 & 0 & 0 & 0 & 0 & 0 & 0 & 0 & 7,9 & 0 & 0 & 0 & 0 & 38,7 & Medio & Media \\
\hline & Ruta del Agua 2 & 6,2 & 5,3 & 10,9 & 8,2 & 8,5 & 0 & 7,6 & 0 & 0 & 0 & 0 & 0 & 7,9 & 0 & 0 & 8,2 & 5,0 & 67,7 & Alto & Media \\
\hline \multirow{2}{*}{$\begin{array}{l}\text { Alta Calidad } \\
\text { y Baja } \\
\text { Preferencia }\end{array}$} & $\begin{array}{c}\text { Capilla San Juan } \\
\text { Bautista }\end{array}$ & 0 & 0 & 0 & 0 & 0 & 0 & 0 & 0 & 1,8 & 3,2 & 3,8 & 0 & 0 & 0 & 0 & 0 & 5,0 & 13,8 & Bajo & Media \\
\hline & $\begin{array}{c}\text { Centro Ceremonial } \\
\text { Xochiquia }\end{array}$ & 0 & 0 & 0 & 0 & 0 & 0 & 0 & 0 & 0 & 3,2 & 0 & 0 & 0 & 0 & 0 & 0 & 0 & 3,2 & Bajo & Media \\
\hline \multirow{2}{*}{$\begin{array}{l}\text { Baja Calidad } \\
\text { y Alta } \\
\text { Preferencia }\end{array}$} & Ruta del Agua 1 & 0 & 0 & 0 & 8,2 & 8,5 & 4,4 & 0 & 0 & 0 & 0 & 0 & 0 & 7,9 & 0 & 0 & 0 & 5,0 & 34,0 & Medio & Baja \\
\hline & $\begin{array}{l}\text { Ruta Poblado } \\
\text { Atlautla-campo }\end{array}$ & 0 & 0 & 0 & 8,2 & 8,5 & 4,4 & 0 & 0 & 0 & 0 & 0 & 0 & 7,9 & 0 & 0 & 0 & 5,0 & 34,0 & Medio & Baja \\
\hline \multirow{2}{*}{$\begin{array}{l}\text { Baja Calidad Y } \\
\text { preferencia }\end{array}$} & Paraje Calpisca & 0 & 0 & 10,9 & 0 & 8,5 & 0 & 0 & 0 & 0 & 0 & 0 & 0 & 0 & 0 & 0 & 0 & 5,0 & 24,3 & Bajo & Baja \\
\hline & $\begin{array}{l}\text { Templo San Juan } \\
\text { Evangelista }\end{array}$ & 0 & 0 & 0 & 0 & 0 & 0 & 0 & 0 & 1,8 & 0 & 3,8 & 0 & 0 & 0 & 0 & 0 & 5,0 & 10,6 & Bajo & Baja \\
\hline
\end{tabular}

Fuente: elaboración propia. 
Figura $\mathrm{N}^{\circ} 2$

Distribución y valoración de la calidad paisajística y preferencia de los 29 atractivos turísticos del municipio de Atlautla, México.

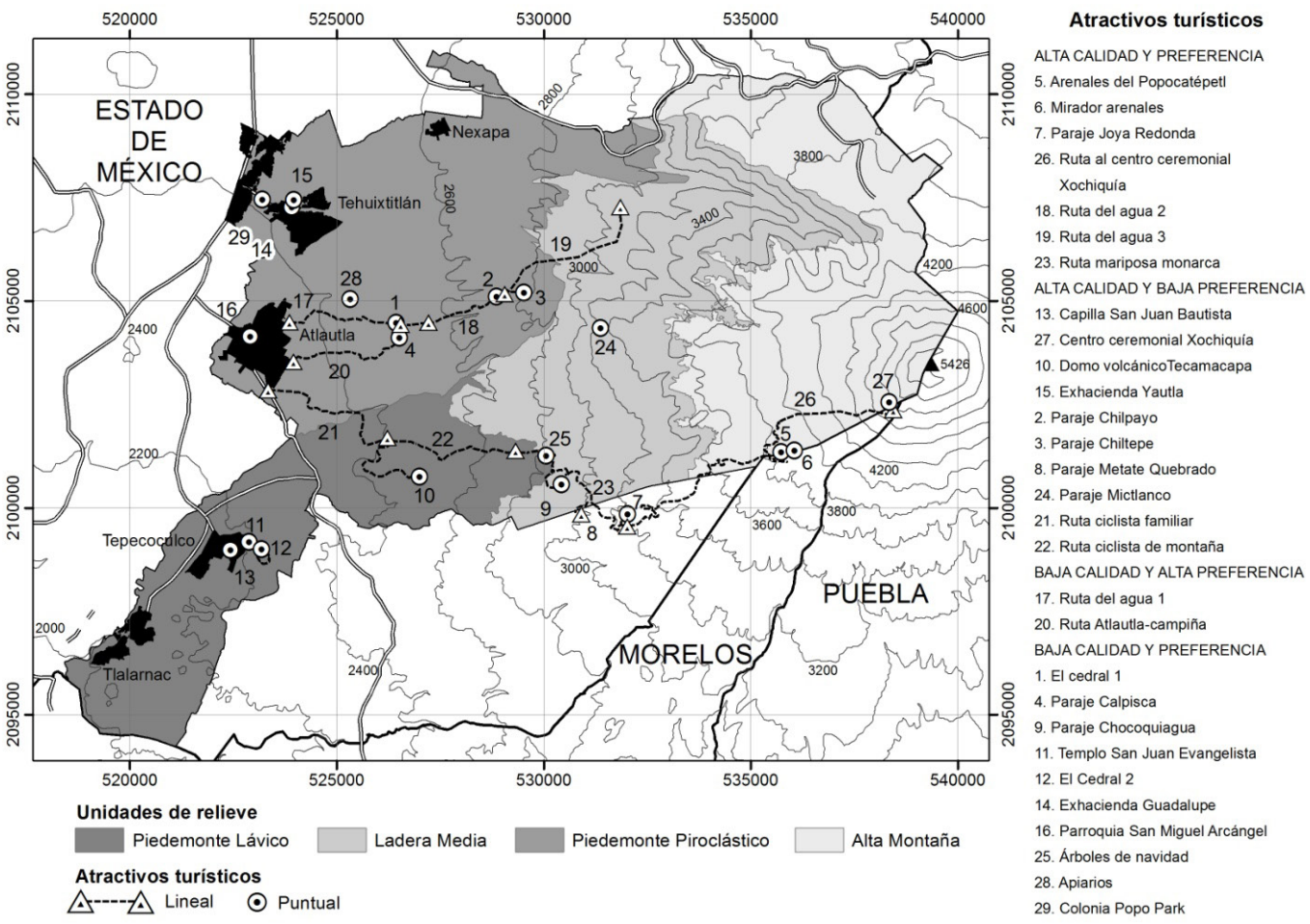

Fuente: Elaboración propia.

\section{Consideraciones finales}

\section{La calidad del paisaje como recurso para el turismo en el medio rural}

En este estudio se parte de considerar que el paisaje sintetiza las condiciones geomorfológicas, bióticas y culturales de un territorio, algunas de las cuales son susceptibles de ser evaluadas como recursos de interés para determinar la calidad de los atractivos turísticos en ambientes rurales (Dosso, 2011; Dos Santos, 2011; Cebrián-Abellán, 2013; Carneiro et al., 2015). Se propone así una lista de 29 indicadores, basados en criterios objetivos y mensurables, para evaluar la calidad de los componentes que definen la compleja estructura del paisaje al interior de los atractivos turísticos (Muñoz, 1998; Cawley \& Gillmor, 2008). De manera complementaria a otros estudios que parten de una perspectiva estética o visual (Zuluaga, 2006; Knudsen et al., 2013; Kalivoda et al., 2014), los indicadores aquí propuestos hacen referencia a tres criterios de valoración del paisaje - escénico, ambiental y cultural -, con los cuales se pretende generar una evaluación completa e integrada, de amplia validez para ser aplicada en otras áreas rurales distintas o contrastadas. 
Nuestra experiencia en Atlautla confirma los resultados obtenidos en trabajos previos, donde se ha encontrado que el uso de indicadores objetivos con un enfoque de paisaje, seleccionados y evaluados con el apoyo de los miembros de la comunidad, facilita la intervención de los actores sociales locales en el diseño de proyectos de desarrollo turístico, condición necesaria para el éxito en la implantación y desarrollo de esta actividad (Barbini, 2005; Serrano, 2008; Nogué et al., 2009; Picazo, 2012).

Encontramos que el área de estudio presenta una gran variedad de recursos turísticos con potencial para cubrir una amplia gama de motivaciones turísticas, principalmente físicas (aventura, descanso, salud, deporte y placer), culturales (educación, entornos, nuevas culturas, gastronomía y medicina tradicional) y religiosas (peregrinaciones, ceremonias religiosas tradicionales), todas las cuales son consistentes con las nuevas modalidades del denominado turismo alternativo (Berry \& Ladkin, 1997; Weaver \& Lawton, 1999). En este sentido, es posible proyectar el aprovechamiento de dichas actividades para el turismo de aventura, agroturismo, turismo místico-religioso y turismo de naturaleza (Torres, 2003; Waitt et al., 2003).

Particularmente, se identificaron diversos atractivos turísticos de alta calidad que pueden funcionar como puntos nodales a partir de los cuales impulsar el aprovechamiento de otros de menor calidad, integrándose todos ellos en una misma cadena de valor, por ejemplo, mediante rutas turísticas que permitan el desarrollo integral de la actividad turística (OMT, 1999; Ryan, 2002), romper con la estacionalidad de la actividad turística, diversificándola territorialmente y generando una mayor productividad y sostenibilidad en los destinos (Nel-lo \& Pérez-Albert, 2003; Cawley \& Gillmor, 2008), y una mayor capacidad para satisfacer, desde el punto de vista de la demanda social, la micro-movilidad de un mayor mercado (Lane \& Kastenholz, 2015), así como ampliar los beneficios de la actividad a un sector más amplio de la comunidad local.

\section{La disociación entre la calidad paisajística y el elemento social}

Como en todo fenómeno turístico, existe para el caso de Atlautla un componente sociocultural complejo y que requiere de una mayor congruencia con sus posibilidades de desarrollo (Tarroja, 2004; Pingel, 2007). Por ejemplo, en la comunidad se tiene una clara percepción del potencial que los recursos paisajísticos aportan al desarrollo del turismo, sobre todo en el caso de los bosques y las montañas que son reconocidos como principales destinos de descanso y recreación. Sin embargo, aunque el interés por desarrollar el turismo involucra a la mayoría de los habitantes, no se observó suficiente claridad en cuanto a las posibilidades de participación en el corto plazo.

Esta situación puede tener relación directa con la cercanía y la influencia de orden económico que la Ciudad de México tiene sobre la región de Atlautla. El proceso de dominación de la ciudad sobre el campo (Vera, 2011), se explica a partir de fenómenos tales como la búsqueda de fuentes de empleo y centros de consumo en la ciudad, el abandono gradual de las labores agrícolas tradicionales, la urbanización y la adopción de nuevos patrones culturales, los que tienen un fuerte impacto en la sociedad atlautlense.

Por otra parte, la disociación entre el potencial turístico y el elemento social puede explicarse por aspectos endógenos. El desarrollo rural basado en expresiones turísticas necesita de una buena dinámica institucional local, en torno a actores locales con una fuerte identidad sociocultural, que sean capaces de generar estrategias de interrelación local/global en busca de beneficios co- 
munes (Mantero, 2004; Barbini, 2005). Sin embargo, nuestros resultados para Atlautla revelan la existencia de graves disputas por la propiedad y el uso de la tierra (Flores \& Rello, 2001), en buena medida provocadas por la falta de líderes capaces de solucionar los conflictos. En consecuencia, prima en la comunidad un bajo nivel de comunicación y colaboración al interior y, por lo tanto, una reducida capacidad organizativa que se constituye como una de las principales limitantes para el adecuado manejo de recursos comunes (Flores \& Rello, 2001).

Frente a esta situación, la comunidad identifica al turismo como una opción viable para revertir la creciente pérdida de interés por las costumbres, tradiciones, herencia histórica y sentido de identidad por parte del sector más joven de la población. También para rescatar algunas manifestaciones histórico-culturales, tales como la medicina tradicional, la conservación de las lenguas nativas, las manifestaciones ceremoniales, místicas o perceptuales de quienes habitan y coexisten con el bosque, la montaña y sus recursos. La implicación de la población local es determinante para el éxito del turismo, pues de una buena base colectiva depende en gran medida la acogida del visitante y la responsabilidad activa en la consolidación de los esquemas locales de organización y la capacidad de autogestión. En particular, la colaboración colectiva representa una oportunidad para atender otro tipo de carencias que existen en cuanto a equipamientos, servicios básicos e infraestructura (Castillo et al., 2009, Lane \& Kastenholz, 2015).

Si, como establece Barbini (2008), la naturaleza y el alcance de las relaciones sociales al interior de las comunidades moldean el desarrollo, resulta entonces prioritario el objetivo de fortalecer el capital social de Atlautla, es decir, el conjunto de valores y normas compartidas que promueven la cooperación social, y que permitirán determinar el desarrollo turístico (Espinoza et al., 2012). En ese sentido los actores sociales deben asimilar la visión de que el aprovechamiento de los recursos naturales y culturales de la comunidad debe constituir un beneficio colectivo a mediano y largo plazos.

En conclusión, los indicadores propuestos aportan una visión integral del paisaje como complejo ecológico, cultural, social y visual, capaz de establecer una valoración aplicada para el desarrollo de actividades económicas alternativas centradas en un turismo sostenible en armonía con las poblaciones locales, que recoge recientes directrices de carácter internacional en expansión derivadas de la aprobación en Europa del Convenio Europeo del Paisaje.

Además, la propuesta aquí presentada puede ser aplicada en otras comunidades rurales de diferente condición, como herramienta de apoyo para la realización de proyectos locales durante la etapa de diagnóstico, así como complementar los enfoques que abordan el turismo desde otros puntos de vista, como el económico, administrativo, legal, etc., y, en particular, aquellos que se basan en la participación directa de las comunidades locales como elemento central para el desarrollo del turismo sostenible.

\section{Referencias bibliográficas}

ALONSO, G.J. El paisaje como recurso turístico: la Sierra del Segura. En: PILLET, F. Cañizares Ruiz, M. y Ruiz Pulpón, A. (coordinadores). Geografía, Territorio y Paisaje: el estado de la cuestión. Actas del XXI Congreso de la Asociación de Geógrafos Españoles. Cuenca: Ediciones de la Universidad de Castilla-La Mancha, 2009, p. 1121-1134. 
BARBINI, B. Viabilidad social para el desarrollo turístico en centros urbanos bonaerenses. Aportes y transferencias, 2005, Vol. 9, N² 2, p. 148-158.

BARBINI, B. Capital social y desarrollo. Aplicación de indicadores de capital social al ámbito turístico. Aportes y transferencias, 2008, Vol. 12, № 2, p. 65-91.

BERRY, S. \& LADKIN, A. Sustainable tourism: A regional perspective. Tourism Management, 1997, Vol. $18, N^{\circ} 7$, p. 433-440.

BERTRAND, C. y BERTRAND, G. Geografía del medio ambiente: el sistema GTP: geosistema, territorio y paisaje. Granada: Universidad de Granada, 2006.

BRUNI, D. Landscape quality and sustainability indicators. Agriculture and Agricultural Science Procedia, 2016, Vol. 8, p. 698-705.

CARNEIRO, M.J.; LIMA, J. \& LAVRADOR, A. Landscape and the rural tourism experience: identifying key elements, addressing potential, and implications for the future. Journal of Sustainable Tourism, 2015, Vol. 23, № 8-9, p. 1217-1235.

CASTILLO, Y.; DÍAZ, M.; HERRERA, E. y ORTIZ M. Creación de Productos Turísticos de Intereses Especiales para la Provincia de Palena, Región de Los Lagos, Chile. Valdivia: Tesis, Escuela de Turismo, Universidad Austral de Chile, 2009.

CASSATELLA, C. Assesing visual and social perceptions of landscape. In: CASSATELLA, C. \& PEANO, A. (editors). Landscape indicators. Assessing and monitoring landscape quality. Springer, 2011, p. 105-140.

CASSATELLA, C. \& PEANO, A. (editors). Landscape indicators. Assessing and monitoring landscape quality. London: Springer, 2011.

CAWLEY, M. \& GILLMOR, D.A. Integrated rural tourism: Concepts and Practice. Annals of Tourism Research, 2008, Vol. 35, № 2, p. 316-337.

CEBALLOS, A. \& LÓPEZ-BLANCO, J. Delineation of suitable areas for crops using a Multi-Criteria Evaluation approach and land use/cover mapping: a case study in Central Mexico. Agricultural Systems, 2003, Vol. 77, N² 2, p. 117-136.

CEBRIÁN-ABELLÁN, F. La función del paisaje como recurso territorial turístico en zonas de interior, Observatorio medioambiental, 2013, Nº 16, p. 37-54.

DOSSO, R.H. Turismo y paisaje: pluralidad conceptual, versatilidad interpretativa y responsabilidad interdisciplinar. Aportes y transferencias. Tiempo Libre. Turismo y Recreación, 2011, № 15, p. $15-54$.

DOS SANTOS, P. Marco teórico-metodológico de los estudios del paisaje: Perspectivas de aplicación en la planificación del turismo. Estudios y Perspectivas en Turismo, 2011, Vol. 20, № 3, p. 522-541. 
ESPINOZA, S.R.; CHÁVEZ, D.R. y ANDRADE, R.E. Lo global-local como base del turismo y la sustentabilidad. Una aproximación a la discusión teórica conceptual". En: LÓPEZ LÓPEZ, A.; LÓPEZ PARDO, G.; ANDRADE ROMO, E.; DAGOSTINO CHÁVEZ, R.M. y ESPINOZA SÁNCHEZ, R. (coordinadores). Lo local y el turismo. Nuevos paradigmas de interpretación. Guadalajara: Academia Mexicana de Investigación Turística A. C., Universidad de Guadalajara, 2012, p. 41-53.

FERNÁNDEZ ROBIN, C.; CEA VALENCIA, J.; SANTANDER, P. y MELO ORREGO R. Turismo de intereses especiales: investigación de mercado sobre las motivaciones desde la perspectiva del cliente. Revista Internacional Administración \& Finanzas, 2015, Vol. 8, №1, p. 51-68.

FLORES, M. y RELLO, F. Capital social. Virtudes y limitaciones. En: Conferencia Regional sobre Capital Social y Pobreza, Santiago de Chile, Chile, 2001. Disponible en Internet: http://www.flacsoandes.edu.ec/sites/default/files/agora/files/1267551205.capital_social.pdf

FRY, G.; TVEIT, M. S.; ODE, A, \& VELARDE, M.D. The ecology of visual landscapes: Exploring the conceptual common ground of visual and ecological landscape indicators. Ecological Indicators, 2009, No 9, p. 933- 947.

GERONTA, CH. The role of landscape in the sustainable planning and management of tourism destinations: the case study of the Island of Rhodes. In: 5th International Scientific Conference "Tourism trends and advances in the 21st century", Rodas, Grecia, 2016. Disponible en Internet: https://www.researchgate.net/publication/272350917

GOTTERO, E. \& CASSATELA, C. Landscape indicators for rural development policies. Application of a core set in the case study of Piedmont Region. Environmental Impact Assessment Review, 2017, No 65, p. 75-85.

GUTIÉRREZ, D. La Construcción de indicadores como problema epistemológico. Cinta Moebio, $2009, N^{\circ} 34$, p. 16-36.

INSTITUTO NACIONAL DE ESTADÍSTICA, GEOGRAFÍA E INFORMÁTICA, INEGI. Prontuario de información geográfica municipal de los Estados Unidos Mexicanos. México: Instituto Nacional de Geografía, Estadística e Informática, 2009.

INSTITUTO NACIONAL PARA EL FEDERALISMO Y EL DESARROLLO MUNICIPAL, INAFED. Enciclopedia de los municipios y delegaciones de México, Municipios del Estado de México, Atlautla. México: Instituto Nacional para el Federalismo y el Desarrollo Municipal, 2018. Disponible en Internet: www.inafed.gob.mx/work/enciclopedia/EMM15mexico/municipios/15015a.html

KALIVODA, O.; VOJAR, J.; SKRIVANOVÁ, Z. \& ZAHRADNÍK, D. Consensus in landscape preference judgments: The effects of landscape visual aesthetic quality and respondents' characteristics. Journal of Environmental Management, 2014, №137, p. 36-44.

KNUDSEN, D.C.; METRO-ROLAND, M.M. \& RICKLY-BOYD, J.M. Landscape studies and tourism research. In: HOWARD, P.; THOMPSON, I. \& WATERTON, E. (editors). The Routledge Companion to Landscape Studies. New York: Routledge, 2013, p. 286-295. 
LANE, B. \& KASTENHOLZ, E. Rural tourism: the evolution of practice and research approaches towards a new generation concept? Journal of Sustainable Tourism, 2015, Vol. 23, № 8-9, p. 11331156.

LEADER-ELLIOTT, L. Cultural landscapes of a tourism destination: South Australia's Barossa Valley. In: Understanding Cultural Landscapes Symposium, Adelaida, Australia, Flinders University, 2005.

MANTERO, J.C. Desarrollo local y actividad turística. Aportes y transferencias, 2004, Vol. 8, № 1 , p. 11-38.

MUÑOZ, J. Paisaje y geosistema. Una aproximación desde la Geografía Física. En: MARTíNEZ DE PISÓN, E. (director). Paisaje y Medio Ambiente. Valladolid: Fundación Duques de Soria, Universidad de Valladolid, 1998, p. 45-55.

MUÑOZ-PEDREROS, A. La evaluación del paisaje: una herramienta de gestión ambiental. Revista Chilena de Historia Natural, 2004, Vol. 77, №1, p. 139-156.

NEL-LO A.M. y PÉREZ-ALBERT Y. Papel de la población local en la actividad ecoturística: los casos de México y Costa Rica. En: MANERO, F. y PASTOR, L.J. (coordinadores). El espacio latinoamericano: cambio económico y gestión urbana en la era de la globalización. Valladolid: Universidad de Valladolid, 2003, p. 207-214.

NOGUÉ, J.; PUIGBERT, L. \& BRETCHA, G. (editores). Indicadors de paisatge. Reptes i perspectives. Barcelona: Olot: Observatori del Paisatge, 2009.

ORGANIZATION FOR ECONOMIC CO-OPERATION AND DEVELOPMENT, OECD. OECD Environmental Indicators: Development, Measurement and Use: Paris, 2003. Disponible en Internet: http:// www.oecd.org/env

ORGANIZACIÓN MUNDIAL DEL TURISMO, OMT. Agenda para Planificadores Locales: Turismo y Gestión Municipal. Madrid: Organización Mundial del Turismo, 1999.

PICAZO, H. ¿Por qué viajamos donde viajamos? Una Introducción a la estrecha relación entre Turismo y Paisaje. Revista Eubacteria, 2012, N² 29, p. 1-5.

PINGEL, C. Turismo solidario en el marco del turismo responsable. Contextos, conceptos, y aplicaciones. Mar del Plata: Monografía de Graduación, Facultad de Ciencias Económicas y Sociales, Universidad Nacional de Mar del Plata. 2007. Disponible en internet: http://nulan.mdp.edu. ar/1406/1/pingel_c.pdf.

PRALONG, J.P. A method for assessing tourist potential and use of geomorphological sites. Géomorphologie: relief, processus, environnement, 2005, Vol. 11, №3, p. 189-196.

REYNARD, E.; FONTANA, G.; KOZLIK, L. \& SCAPOZZA, C. A method for assessing «scientific» and «additional values» of geomorphosites. Geographica Helvetica, 2007, Vol. 62, № 3, p. 148-158. 
RYAN, C. Equity, management, power sharing and sustainability: issues of the new tourism. Tourism Management, 2002, $\mathrm{N}^{\circ}$ 23, p. 17-26.

SALINAS, E.; NAVARRO, E.; ECHARRI, M. y La O, J.A. Metodología para la evaluación de la sustentabilidad territorial: el uso de indicadores en destinos turísticos de Cuba. Boletín de la Real Sociedad Geográfica, 2008, №144, p. 77-102.

SERRANO, D. Ensayo metodológico para la valoración estética del paisaje. Aplicación en Muntanyes D'Ordal, Barcelona. Geographicalia, 2008, N 54, p. 99-112.

SOWIŃSKA-ŚWIERKOSZ B.N. \& CHMIELEWSKI T.J. A new approach to the identification of Landscape Quality Objectives (LQOs) as a set of indicators. Journal of Environmental Management, 2016, No 186, p. 596-608.

TARROJA, A. Paisaje y gestión del territorio: transformaciones territoriales y valoración social del paisaje, Monográfico sobre paisaje y gestión del territorio. Madrid: Colegio de Geógrafos, 2004, p.1-8.

TORRES, R. Linkages between tourism and agriculture in México. Annals of Tourism Research, 2003, Vol., 30, N³, p. 546-566.

UUEMAA, E.; MANDER, U. \& MARJA, R. Trends in the use of landscape spatial metrics as landscape indicators: A review. Ecological Indicators, 2013, № 28, p.100-106.

VERA, R.J.F. (coordinador). Análisis territorial del turismo y planificación de destinos turísticos: Valencia: Tirant lo Blanch, 2011.

WAITT, G.; LANE, R. \& HEAD, L. The boundaries of nature tourism. Annals of Tourism Research, 2003, Vol. 30, No 3, p. 523-545.

WEAVER, D. \& LAWTON, L. Sustainable tourism. A critical analysis. Queensland: CRC for sustainable tourism Pty. Ltd, 1999. Disponible en Internet:

http://citeseerx.ist.psu.edu/viewdoc/download; jsessionid=5A3 A53ED48F7D1A46C393A16E3E2043E?doi=10.1.1.606.327\&rep=rep1\&type=pdf

ZULUAGA, P.A. Una mirada al paisaje como recurso turístico. Revista Interamericana de Ambiente y Turismo, 2006, Vol. 2, N² 2, p. 76-82. 
